\title{
Chronic lymphocytic inflammation with pontine perivascular enhancement responsive to steroids (CLIPPERS) after SARS-CoV-2 pneumonia
}

\author{
Federico Montini ${ }^{1,2}$ (D) Vittorio Martinelli ${ }^{1}$ [D $\cdot$ Francesca Sangalli $^{1} \cdot$ Marcella Callea $^{3} \cdot$ Nicoletta Anzalone $^{2,4}$. \\ Massimo Filippi ${ }^{1,2,5,6,7}$ (1)
}

Received: 6 May 2021 / Accepted: 7 July 2021 / Published online: 17 July 2021

(c) Fondazione Società Italiana di Neurologia 2021

\section{Introduction}

SARS-CoV-2 is known to cause Coronavirus Disease 2019 (COVID-19), which has extremely variable clinical manifestations ranging from asymptomatic carriers to seriously ill patients with fever, breathing difficulty, acute respiratory distress, and multiple organ complications, eventually leading to death.

Chronic lymphocytic inflammation with pontine perivascular enhancement responsive to steroids (CLIPPERS) is an inflammatory central nervous system (CNS) disorder, prominently involving the brainstem and, more importantly, the pons with variable supratentorial or spinal cord involvement with immune-mediated or other inflammatory pathogenesis [1].

Massimo Filippi

filippi.massimo@hsr.it

1 Neurology Unit, IRCCS San Raffaele Scientific Institute, Via Olgettina, 60, 20132 Milan, Italy

2 Vita-Salute San Raffaele University, Milan, Italy

3 Neuropathology Unit, IRCCS San Raffaele Scientific Institute, Milan, Italy

4 Neuroradiology Unit, IRCCS San Raffaele Scientific Institute, Milan, Italy

5 Neurorehabilitation Unit, IRCCS San Raffaele Scientific Institute, Via Olgettina, 60, 20132 Milan, Italy

6 Neurophysiology Service, IRCCS San Raffaele Scientific Institute, Via Olgettina, 60, 20132 Milan, Italy

7 Neuroimaging Research Unit, Division of Neuroscience, IRCCS San Raffaele Scientific Institute, Via Olgettina, 60, 20132 Milan, Italy

\section{Case report}

A 71-year-old man with no previous medical history was diagnosed with COVID-19 pneumonia in March 2020. Chest computed tomography (CT) indicated mild bilateral interstitial pneumonia, and real-time polymerase chain reaction on nasopharyngeal swab for SARSCoV-2 returned positive. The patient needed no oxygen therapy. He was treated with hydroxychloroquine, azithromycin, and cefexime. The infection outcome was good, and the patient had a full recovery without sequels in a month.

Three months later, the patient sought medical attention for a subacute onset of diplopia in the left gaze, general malaise, and fatigue. Neurological evaluation revealed diplopia in the left gaze with left abducens nerve deficit but no other extraocular muscle deficits or other focal signs. Noncontrast head CT was unremarkable, while brain MRI was characterized by bilateral fluid-attenuated inversion recovery (FLAIR) hyperintensities with punctate, perivascular, and confluent post-gadolinium enhancement in the pons, mesencephalon, hypothalamus, internal capsules, and right hippocampus (Fig. 1).

Cerebrospinal fluid (CSF) analysis was normal (2 cells/ $\mu \mathrm{L}$ ), with no evidence of oligoclonal bands or atypical cells. The screening panel for autoimmune and infectious aetiologies was negative. Epstein-Barr virus viral capsid antigen IgG and IgM antibodies were negative on serum. Wholebody contrast-enhanced CT was unremarkable. Partial spontaneous remission of symptoms occurred within few weeks, persisting only mild fatigue but no diplopia or general malaise. The patient was referred for a stereotactic temporal lobe brain biopsy, which showed non-specific chronic perivascular lymphocytic inflammation (Fig. 2). No therapy was prescribed. 
Fig. 1 Pre-treatment brain MRI was characterized by bilateral fluid-attenuated inversion recovery (FLAIR) hyperintensities in the pons, mesencephalon, hypothalamus, internal capsules, and right hippocampus (A) with punctate, peppering, perivascular, and confluent post-gadolinium enhancement in these sites (B-C-D). After treatment, brain MRI demonstrated partial regression of FLAIR hyperintensities (E) with almost disappearance of post-gadolinium enhancement (F-G-H)

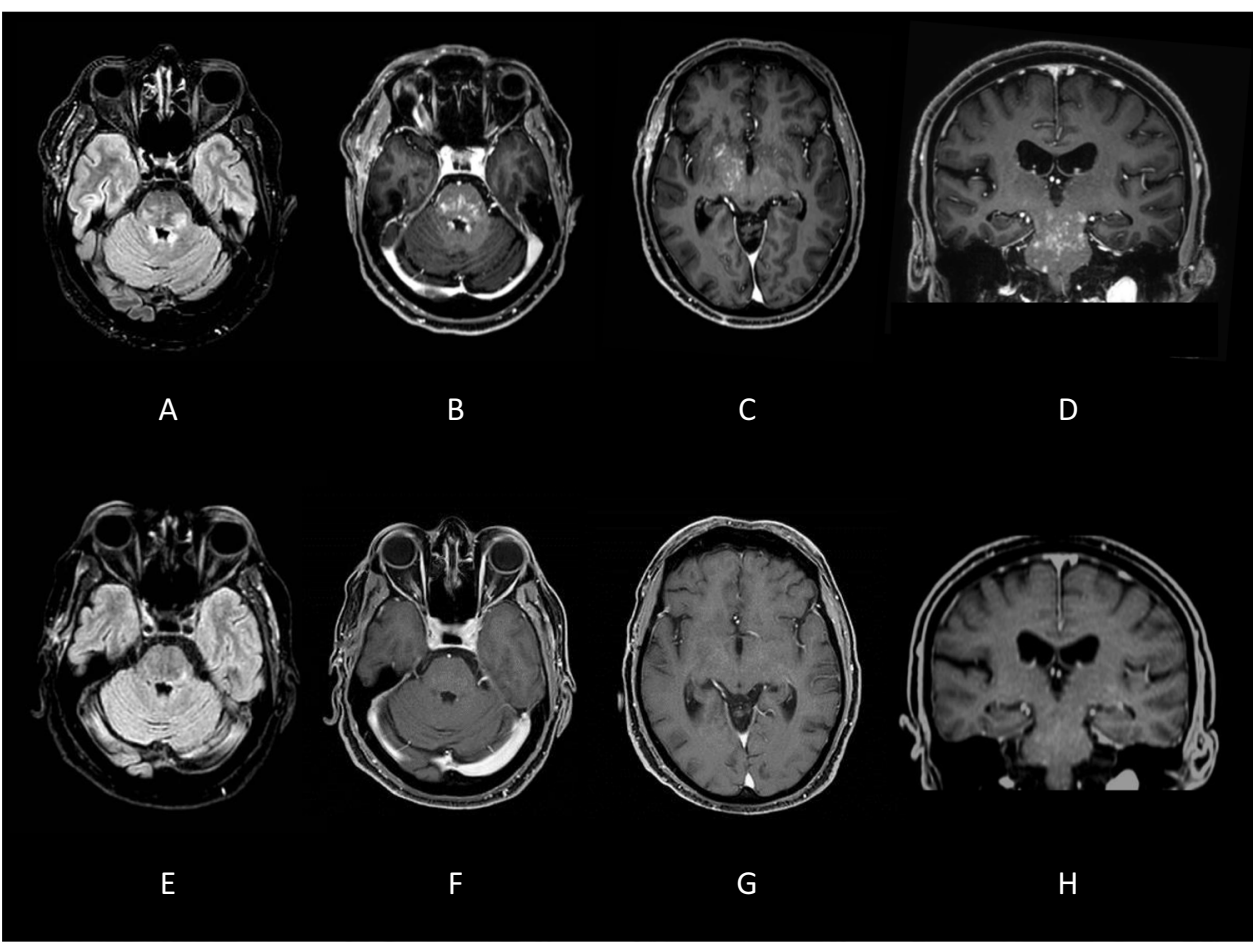

At three-month follow-up, the patient felt well, reporting only mild fatigue. Clinical examination showed bilateral distal leg apallesthesia, and mild left leg drift on the Mingazzini maneuver, which the patient was not aware of. A new brain MRI demonstrated an extension of FLAIR hyperintensities with punctate, perivascular, and confluent post-gadolinium enhancement, especially in the pons. He was treated with intravenous high-dose methylprednisolone with almost complete enhancement regression on MRI and then with daily oral steroid tapering and monthly cycles of intravenous cyclophosphamide with persistent clinical and neuroradiological stability.
Fig. 2 A, B, C Histological slides of formalin-fixed paraffinembedded (FFPE) tumor specimen haematoxylin and eosin (H\&E) stained (A, B 40×, C $20 \times$ ). Figures show brain tissue with inflammatory cell infiltration made by small lymphocytes mostly with perivascular arrangement. D-G Histological slides of FFPE tumor sample immunostained with anti-CD3 $(\mathbf{D}, \mathbf{F}, \mathbf{G})$ and anti-CD20 (E) antibodies. Inflammatory cell component is made by small $\mathrm{T}$ and $\mathrm{B}$ lymphocytes equally represented. They show perivascular distribution $(\mathbf{F}, \mathbf{G})$ but they are also present as isolated or as small intraparenchymal clusters (D, E)

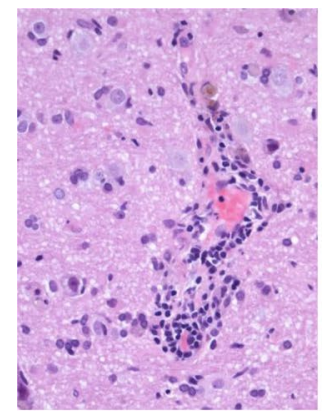

A

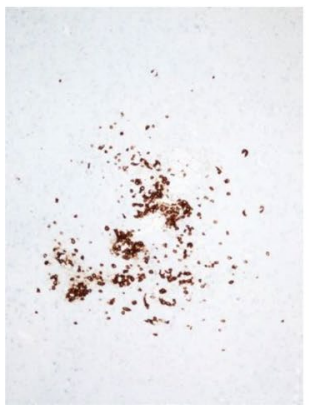

E

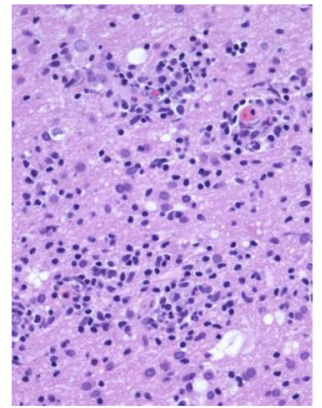

B

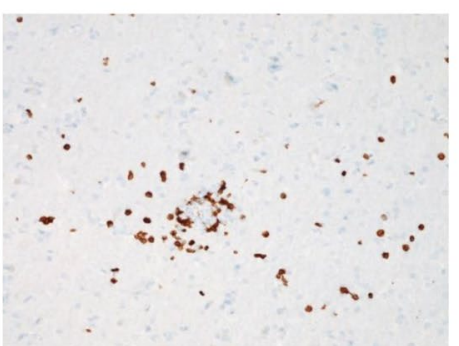

$\mathbf{F}$

C
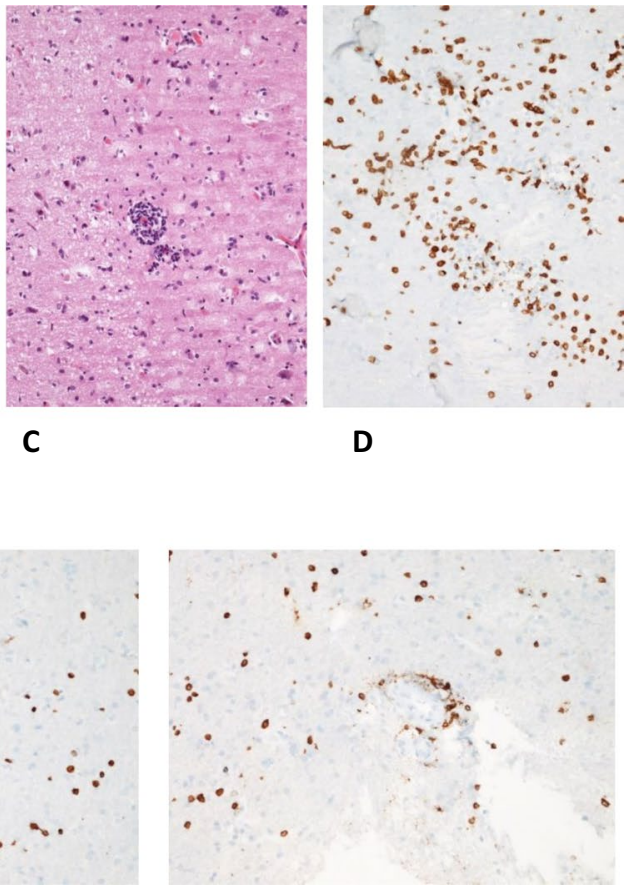

G

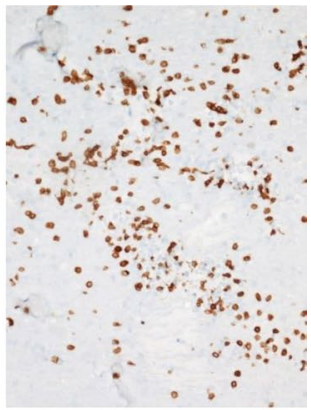

D 
Collected data were suggestive of CLIPPERS with diffuse bilateral supratentorial involvement.

\section{Discussion}

Apart from respiratory symptoms, COVID-19 can be associated with a wide array of neurological manifestations, involving the central and the peripheral nervous systems [2].

Neurological involvement depends on direct cytopathic effects mediated by SARS-CoV-2 infection and the indirect effects secondary to the infection-related inflammatory response [3]. Neuropathologic findings in patients with SARSCoV-2 infection include hypoxic mechanisms, endovascular microthrombi, microbleeds, necrosis, diffuse cerebral edema, gliosis with diffuse activation of microglia and astrocytes, causing various stages of inflammation with cellular components infiltrating into perivascular areas of the brain [3].

In reaction to SARS-CoV-2 infection, the immune system can produce high cytokine levels [4], leading to a hyperinflammatory response and T-cell activation [4]. Such an inflammatory response can cause blood-brain barrier damage as well. CSF analysis of COVID-19 patients [5] showed differentiated monocytes and exhausted T-cells with consequent broad clonal T-cell expansion.

CLIPPERS is a T-cell-mediated disorder with an infiltrate characterized by an abundance of CD4+ T-cell infiltration with less CD8+ and CD20+ cells, without demyelination $[6,7]$ with relative widespread perivascular inflammation and postinflammatory axonal injury [7]. T-cell broad clonal expansion is present in CLIPPERS disease.

CLIPPERS aetiology is unknown. Autoimmunity development is multifactorial and complex, requiring time to evolve [8]. Post-infectious phenomena tend to occur usually few weeks following infection, but they can become clinically evident months or years after the triggering event [8].

A several months delayed autoimmune disease onset has already been described in different conditions, among the others CLIPPERS [9] and Mystenia Gravis [10].

The overall process leading to this condition may have been triggered by COVID-19 infection in our 71-year-old patient, without any previous immunological disease.

CLIPPERS is so far considered an idiopathic rare disease. This is the first report after COVID-19 disease with a diffuse involvement of brainstem and supratentorial regions. Even though a single case is not enough to suggest a causal link, future reports could support this association.

\section{Declarations}

Ethical approval This article does not contain any studies with human participants or animals performed by any of the authors.
Conflict of interest F Montini, M Callea, N Anzalone have nothing to disclose.

V Martinelli received honoraria for consulting services or speaking activity from Biogen, Merck, Novartis, TEVA, Almirall, and SanofiGenzyme.

F Sangalli received travel founding and/or speaker honoraria from Merk, Biogen, Novartis and Sanofi-Genzyme.

M. Filippi is Editor-in-Chief of the Journal of Neurology, Associate Editor of Human Brain Mapping, Associate Editor of Radiology, and Associate Editor of Neurological Sciences; received compensation for consulting services and/or speaking activities from Alexion, Almirall, Bayer, Biogen, Celgene, Eli Lilly, Genzyme, Merck-Serono, Novartis, Roche, Sanofi, Takeda, and Teva Pharmaceutical Industries; and receives research support from Biogen Idec, Merck-Serono, Novartis, Roche, Teva Pharmaceutical Industries, Italian Ministry of Health, Fondazione Italiana Sclerosi Multipla, and ARiSLA (Fondazione Italiana di Ricerca per la SLA).

\section{References}

1. Dudesek A, Rimmele F, Tesar S, Kolbaske S, Rommer PS, Benecke R et al (2014) CLIPPERS: chronic lymphocytic inflammation with pontine perivascular enhancement responsive to steroids. Review of an increasingly recognized entity within the spectrum of inflammatory central nervous system disorders. Clin Exp Immunol. 175(3):385-96

2. Ellul MA, Benjamin L, Singh B, Lant S, Michael BD, Easton A et al (2020) Neurological associations of COVID-19. The Lancet Neurology 19(9):767-783

3. Pajo AT, Espiritu AI, Apor A, Jamora RDG (2021) Neuropathologic findings of patients with COVID-19: a systematic review. Neurol Sci 42(4):1255-1266

4. Bhaskar S, Sinha A, Banach M, Mittoo S, Weissert R, Kass JS et al (2020) Cytokine storm in COVID-19-immunopathological mechanisms, clinical considerations, and therapeutic approaches: the REPROGRAM Consortium Position Paper. Front Immunol 11:1648

5. Heming M, Li X, Rauber S, Mausberg AK, Borsch AL, Hartlehnert $\mathrm{M}$ et al (2021) Neurological manifestations of COVID-19 feature $\mathrm{T}$ cell exhaustion and dedifferentiated monocytes in cerebrospinal fluid. Immunity. 54(1):164-75e6

6. Pittock SJ, Debruyne J, Krecke KN, Giannini C, van den Ameele J, De Herdt V et al (2010) Chronic lymphocytic inflammation with pontine perivascular enhancement responsive to steroids (CLIPPERS). Brain 133(9):2626-2634

7. Blaabjerg M, Ruprecht K, Sinnecker T, Kondziella D, Niendorf T, Kerrn-Jespersen BM et al (2016) Widespread inflammation in CLIPPERS syndrome indicated by autopsy and ultra-high-field 7T MRI. Neurol Neuroimmunol Neuroinflamm. 3(3):e226

8. Getts DR, Chastain EM, Terry RL, Miller SD (2013) Virus infection, antiviral immunity, and autoimmunity. Immunol Rev 255(1):197-209

9. Wang L, Holthaus EA, Jimenez XF, Tavee J, Li Y (2015) MRI evolution of CLIPPERS syndrome following herpes zoster infection. J Neurol Sci 348(1-2):277-278

10. Leis AA, Szatmary G, Ross MA, Stokic DS (2014) West nile virus infection and myasthenia gravis. Muscle Nerve 49(1):26-29

11. Solomon IH, Normandin E, Bhattacharyya S, Mukerji SS, Keller K, Ali AS et al (2020) Neuropathological features of Covid-19. N Engl J Med 383(10):989-992

Publisher's note Springer Nature remains neutral with regard to jurisdictional claims in published maps and institutional affiliations. 